\title{
A universal power law for metallic glasses
}

\author{
M.Q. Jiang, ${ }^{\mathrm{a}, \mathrm{b}, *}$ G. Wilde, ${ }^{\mathrm{b}}$ J.B. Gao ${ }^{\mathrm{c}}$ and L.H. Dai ${ }^{\mathrm{a}, *}$ \\ ${ }^{a}$ State Key Laboratory of Nonlinear Mechanics, Institute of Mechanics, Chinese Academy of Sciences, Beisihuanxi Road 15, \\ Beijing 100190, People's Republic of China \\ ${ }^{\mathrm{b}}$ Institute of Materials Physics, Westfälische Wilhelms-Universität Münster, Wilhelm-Klemm Strasse 10, Münster 48149, Germany \\ ${ }^{\mathrm{c}}$ Department of Mechanical and Materials Engineering, Wright State University, Dayton, OH 45435, USA
}

Received 12 July 2013; revised 26 August 2013; accepted 26 August 2013

Available online 3 September 2013

\begin{abstract}
We report a universal power law between bulk modulus and molar volume that holds for metallic glasses (MGs) and most polycrystalline metals, which demonstrates that the volumetric derivative of energy minima can be determined by the average atomic volume. Our findings reveal a characteristic size rang of the short-range order of $0.23-0.32 \mathrm{~nm}$ in MGs that is closest to that in face-centered cubic metals. More interestingly, the short-range order in noncrystalline and crystalline metals seem to be self-similar from a viewpoint of the compressibility.
\end{abstract}

(C) 2013 Acta Materialia Inc. Published by Elsevier Ltd. All rights reserved.

Keywords: Metallic glass; Bulk modulus; Molar volume; Power law

The emergence of metallic glasses (MGs) poses large challenges to our knowledge of conventional glasses and crystalline metals [1-3]. The deviation of the atomic configuration from its crystalline counterpart causes metallic glasses to be in a thermodynamically metastable state, which corresponds to a local minimum of a very complicated potential energy landscape (PEL) [4-6]. The depth of the potential well reflects the stability of the system, and the landscape curvature near its minimum determines the system's elastic properties. Searching for the stability-elasticity correlations is a hot topic in condensed matter physics and materials science [7-9], since it opens a window onto the understanding of the structure-property relationship of matter. The stability-elasticity correlation in PEL implies that mechanical instabilities for a system can be governed by its elastic constants. For isotropic solids such as MGs and polycrystalline metals, there are only two independent elastic constants, namely, shear modulus and bulk modulus. The shear modulus measures the intrinsic capability of the PEL to resist distortion, while the bulk modulus measures the inverse compressibility of the

\footnotetext{
* Corresponding authors. Address: State Key Laboratory of Nonlinear Mechanics, Institute of Mechanics, Chinese Academy of Sciences, Beisihuanxi Road 15, Beijing 100190, People's Republic of China. Tel.: +86 10 52843931; fax: +86 1082543977 (M.Q. Jiang); e-mail addresses: mqjiang@imech.ac.cn; 1hdai@Inm.imech.ac.cn
}

system. Many models [7,9-11] reveal that the barrier height for plastic instability or viscous flow of MGs to occur is proportional to the shear modulus, which is strongly configuration dependent. In contrast, the bulk modulus seems to be insensitive to the subtle structural details [12-14], which possibly implies a universal characteristic of the structures of MGs. In this paper, we theoretically derived a power-law relationship between bulk modulus and molar volume for isotropic solids that universally holds not only for metallic glasses but also for most polycrystalline metals. The physics behind the relation is briefly discussed as well.

In seeking for a relation between the bulk modulus and stability of a system, we consider the potential energy for one molar atom of an MG. Previous studies have shown that the model system of a LennardJones-type interatomic interaction function could predict precisely the lattice parameters for intermetallic systems [15-17], and could even be representative of more complex glasses [18]. In that case, the potential energy has the simplest landscape, given as the following function:

$U=\frac{1}{2} N_{A} \cdot 4 \varepsilon\left[-\left(\frac{\sigma}{r}\right)^{m}+\left(\frac{\sigma}{r}\right)^{n}\right]$

where $N_{A}$ is Avogadro's constant, $\varepsilon$ is the depth of the potential well, $\sigma$ is the finite distance at which the inter-atom potential is zero, $r$ is the distance between two atoms, which is approximately equal to the atomic 
radius, and $m$ and $n$ are the exponents denoting the energy of attraction and repulsion, respectively. Since the average atomic volume scales with the radius $V_{a} \propto r^{3}$, we can rewrite the landscape in terms of the molar volume $\tilde{V}_{m}=N_{A} V_{a}$ with the form:

$U=-\frac{A}{\tilde{V}_{\mathrm{m}}^{m / 3}}+\frac{B}{\tilde{V}_{\mathrm{m}}^{n / 3}}$,

where $A=N_{A}^{1+m / 3} \cdot 2 \varepsilon \sigma^{m}$ and $B=N_{A}^{1+n / 3} \cdot 2 \varepsilon \sigma^{n}$. A system at temperatures well below the glass transition temperature is at or near a potential minimum $U_{0}$, which can be determined by $\partial U_{0} / \partial V_{m}=0$. From this, we obtain:

$U_{0}=\frac{m-n}{n} \frac{A}{V_{m}^{m / 3}}$.

The application of an external pressure will induce a deviation of $\tilde{V}_{m}$ from the equilibrium $V_{m}$. Equating the energy produced by the external pressure to the change in potential energy gives Grüneisen's first rule [19]:

$K=\left.V_{\mathrm{m}} \frac{\partial^{2} U}{\partial \tilde{V}_{\mathrm{m}}^{2}}\right|_{V_{\mathrm{m}}}=\frac{m n}{9} \frac{U_{0}^{\prime}}{V_{\mathrm{m}}}$.

This equation indicates that the bulk modulus at or near equilibrium is a function of the molar volume density of the atomic bonding energy $U_{0}^{\prime}=-U_{0}[20]$. By eliminating $U_{0}^{\prime}$ from Eqs. (3) and (4), and writing $C=10^{3-2 m} \cdot 2 m(n-m) N_{A}^{1+m / 3} \varepsilon \sigma^{m} / 9$, one obtains:

$K V_{\mathrm{m}}^{1+m / 3}=C$,

which represents a power-law relationship between the bulk modulus and the molar volume (equivalently average atomic volume), if the parameter $C$ is constant for the materials considered. Here, $K$ is in units of $\mathrm{GPa}$, and the units of $V_{m}$ are $\mathrm{cm}^{3} \mathrm{~mol}^{-1}$.

Next, we apply the power law (5) to MGs. We collected data of about 80 MGs (Au-, $\mathrm{Ca}-, \mathrm{Cu}-$, rare earth (RE)-, Ni-, Pd-, Pt- and Zr-based) for which the $K$ and $V_{m}$ values were available (Supplementary Table 1). It should be pointed out that these MGs are mainly metalloid free or metallic bond dominated, which guarantees the validity of Eq. (1). For comparison, 55 polycrystalline metals, including 14 face-centered cubic (fcc), 14 body-centered cubic (bcc) and 27 hexagonal closepacked (hcp) lattices, are also considered (Supplementary Table 2). Figure 1 clearly shows a strong correlation between $K$ and $V_{m}$ for all metallic materials regardless of whether they are crystalline or noncrystalline: the smaller the molar volume, the higher the bulk modulus; that is, the more strongly the system resists the bulk deformation. These experimental data can be fitted well by Eq. (5) so long as $m=3.9$ and $C=2.5 \times 10^{4}$. This means that, for materials with similar atomic bond types, their bulk modulus can be fully determined by the molar volume that measures the atomic packing density. To confirm this, we further apply Eq. (5) to a polycrystalline $\mathrm{Fe}-\mathrm{Ti}$ alloy and find that it is also valid for the data if $C$ is adjusted to $C=4.7 \times 10^{4}$ (Supplementary Fig. 1). The inset in Figure 1 magnifies the case for MGs. It is interesting to find that, with increasing bulk modulus and decreasing molar volume, the MG systems tend to be more plastic. This indicates that the

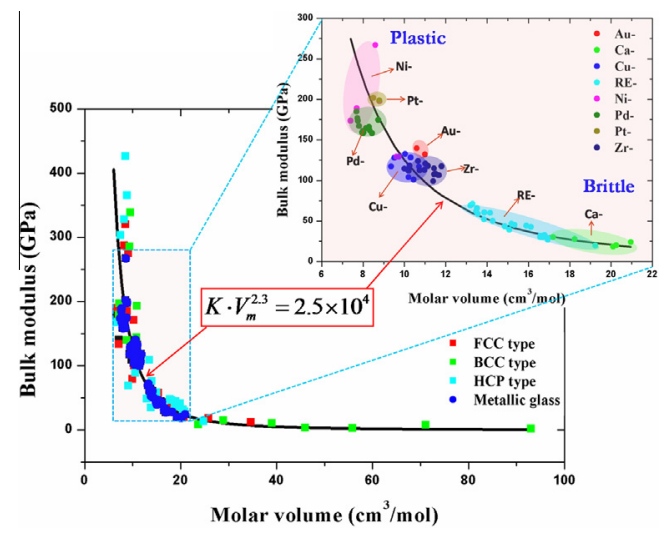

Figure 1. Plot of the bulk modulus vs. the molar volume for about 80 metallic glasses, including $\mathrm{Au}-, \mathrm{Ca}-, \mathrm{Cu}-, \mathrm{RE}-, \mathrm{Ni}-, \mathrm{Pd}-, \mathrm{Pt}-$ and $\mathrm{Zr}-$ based systems, as well as 55 polycrystalline metals ( $14 \mathrm{fcc}, 14 \mathrm{bcc}$ and $27 \mathrm{hcp}$ lattice). The solid line represents the theoretical prediction by Eq. (5). The inset shows the case of metallic glasses, which indicates a brittle-plastic transition with decreasing molar volume and increasing bulk modulus.

atomic packing density might be a key factor to the plasticity of glasses [21-24]. In fact, Rouxel et al. [23] reported a direct correlation between the atomic packing density and Poisson's ratio, which is considered to be a good indicator of a material's plasticity [25-27].

More importantly, the universality of Eq. (5) for both MGs and crystalline metals could provide some insight into their atomic structures. We reconstruct Figure 1 in double-logarithmic coordinates, as shown in Figure 2, where the power law (5) is transformed into a linear dependence. From the viewpoint of the fractal behavior, the correlation shown in Figure 2 is conceptually surprising. It indicates that, for isotropic metals, regardless of their crystalline or glassy state, their bulk modulus or compressibility is self-similar; the value depends on a scaling quantity, which in this case is given by the molar volume. It has been known that MGs preserve short- to medium-range order similar to that in crystalline structures [28-31], although the long-range order is absent. The fractal-like behavior observed here should originate from the short-range order (SRO) structural similarity

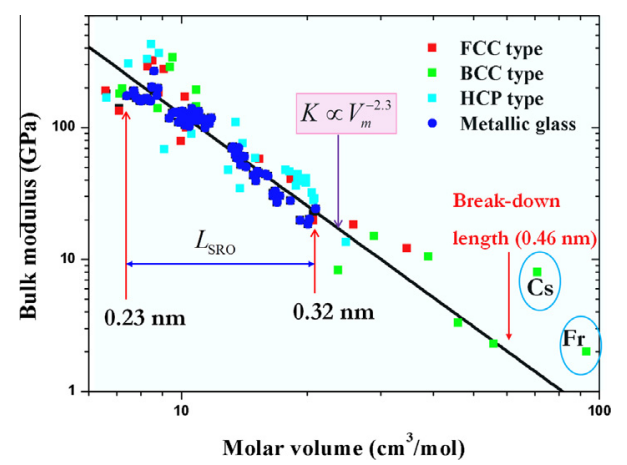

Figure 2. Power law of the bulk modulus vs. the molar volume for a variety of isotropic metal systems shown as double-logarithmic coordinates. The experimental data reveals a short-range order of about $0.23-0.32 \mathrm{~nm}$ for metallic glasses and also demonstrates a critical length of $0.46 \mathrm{~nm}$, beyond which the power law breaks down (e.g. bcc-type Cs and Fr metals). 
among glassy or crystalline metals. This revelation may help explain why nanocrystal formation is often observed in a severely plasticity-localized zone or shear band [32-34]. It is naturally expected that, if the SRO is very similar to the crystalline structure, only a shear transformation could be sufficient to transform a shortto medium-range order region into a critical nucleus for crystallization. In particular, we note that the data of metallic glasses and fcc metals largely overlap. This implies that MGs have the SRO closest to that of fcc metals $[28,29]$, obeying the ideal-mixing rule. From our observations, a characteristic length of the SRO of MGs, $\mathrm{L}_{\mathrm{SRO}}$, of $0.23-0.32 \mathrm{~nm}$ can be identified, corresponding to $7-20 \mathrm{~cm}^{3} \mathrm{~mol}^{-1}$ of molar volume, which is in fair agreement with the measured range of $0.21-$ $0.29 \mathrm{~nm}[30,35]$ and less than the $0.36 \mathrm{~nm}$ reported by direct observation [36]. We also note that two points for the bcc Cs and Fr metals, circled in Figure 2, deviate seriously from the theoretical prediction. This demonstrates that there possibly exists a length scale over which such a size-dependent self-similarity will break down. As indicated in Figure 2, the critical length scale is about $0.46 \mathrm{~nm}$; interestingly, this value is close to the predicted scale $(0.4 \mathrm{~nm})$ for fcc $\mathrm{Cu}$ below which the size dependence of the elasticity should be considered [37].

Finally, it is worth discussing the implications of our present results. The molar volume (or the atomic packing density) reflects the average free volume of MGs to a certain extent. In fact, the free-volume approach [38-40] is very successful as a simple one-parameter description of the "static" structures and their flow instability of glasses. In contrast, it would be expected that a description based on a simple packing density approach would break down if dynamic properties (relaxation dynamics in frequency spaces) were considered, since the dynamics depends directly on the details of the local binding forces and their anisotropy [5-6]. The present findings also indicate that the "nanoglasses" advocated by Gleiter [41] are truly a different state of matter, since their high atomic volume directly means that close packing is not an issue and that a change in the relative contributions of the directional and non-directional binding forces is probably involved.

In summary, the bulk modulus of isotropic atomic bonding systems with densely packed atoms can increase with decreasing average atomic volume, corresponding to a smaller molar volume. The molar volume and the bulk modulus show a universal power-law relationship, which does not depend on how atoms are packed on the long-range scale but originates from the self-similarity of the short-range order under pressure. It should be noted that our current model is essentially a hard-sphere model of atoms for metallic glasses where the electron contributions are not taken into account [42]. This implies that the compressibility of systems at or near equilibrium state can be characterized well by the hard-sphere model [43-45]. However, the compressibility of a system far from an equilibrium state, e.g. under ultrahigh pressure, can be govern by special electron structures. For example, the delocalization of $4 f$ electrons of the constituent $\mathrm{Ce}$ atom under high pressure can contribute to the greater compressibility of $\mathrm{Ce}-\mathrm{Al}$ metallic glasses, causing a transition from a low-bulk-modulus state to a highbulk-modulus state [46].

The work is supported by the NSFC (Nos. 11002144, 11372315, 11132011 and 11023001). M.G.J. acknowledges the Alexander von Humboldt Foundation for support with a post-doctoral fellowship.

Supplementary data associated with this article can be found, in the online version, at http://dx.doi.org/ 10.1016/j.scriptamat.2013.08.022.

[1] W.I. Klement, R.H. Willens, P. Duwez, Nature 187 (1960) 869.

[2] A.L. Greer, Science 267 (1995) 1947.

[3] W.L. Johnson, MRS Bull. 24 (1999) 42.

[4] F.H. Stillinger, Science 267 (1995) 1935.

[5] C.A. Angell, Nature 393 (1998) 521.

[6] P.G. Debenedetti, P.H. Stillinger, Nature 410 (2001) 259.

[7] J.C. Dyre, Rev. Mod. Phys. 78 (2006) 953.

[8] S.B. Maisel, M. Hofler, S. Muller, Nature 491 (2012) 743.

[9] W.H. Wang, Prog. Mater. Sci. 57 (2012) 487.

[10] W.L. Johnson, K. Samwer, Phys. Rev. Lett. 95 (2005) 195501.

[11] B. Zhang, H.Y. Bai, R.J. Wang, Y. Wu, W.H. Wang, Phys. Rev. B 76 (2007) 012201.

[12] G. Duan, M.L. Lind, M.D. Demetriou, W.L. Johnson, W.A. Goddard, T. Cagin, K. Samwer, Appl. Phys. Lett. 89 (2006) 151901.

[13] Y.Q. Cheng, A.J. Cao, E. Ma, Acta Mater. 57 (2009) 3253.

[14] M.Q. Jiang, L.H. Dai, Phys. Rev. B 76 (2007) 054204.

[15] R. Furth, Proc. R. Soc. Lond. A 183 (1944) 87.

[16] A.C. Lund, C.A. Schuh, Acta Mater. 51 (2003) 5399.

[17] E.S. Machlin, Acta. Metall. 22 (1974) 95.

[18] A. Heuer, H.W. Spiess, J. Non-Cryst. Solids 176 (1994) 294.

[19] C. Zwikker, Physical Properties of Solid Materials, Pregamon Press, London, 1954.

[20] T. Rouxel, J. Am. Ceram. Soc. 90 (2007) 3019.

[21] M.Q. Jiang, L.H. Dai, Philos. Mag. Lett. 90 (2010) 269.

[22] J.Q. Wang, W.H. Wang, H.B. Yu, H.Y. Bai, Appl. Phys. Lett. 94 (2009) 121904.

[23] T. Rouxel, H. Ji, T. Hammouda, M. A, Phys. Rev. Lett. $100(2008) 225501$.

[24] O.V. Kuzmin, Y.T. Pei, C.Q. Chen, J.T.M. De Hosson, Acta Mater. 60 (2012) 889.

[25] J.J. Lewandowski, W.H. Wang, A.L. Greer, Philos. Mag. Lett. 85 (2005) 77.

[26] G.N. Greaves, A.L. Greer, R.S. Lakes, T. Rouxel, Nat. Mater. 10 (2011) 823.

[27] S.J. Poon, A.W. Zhu, G.J. Shiflet, Appl. Phys. Lett. 92 (2008).

[28] D.B. Miracle, Nat. Mater. 3 (2004) 697.

[29] H.W. Sheng, W.K. Luo, F.M. Alamgir, J.M. Bai, E. Ma, Nature 439 (2006) 419.

[30] D. Ma, A.D. Stoica, X.-L. Wang, Nat. Mater. 8 (2009) 30.

[31] I. Martin, T. Ohkubo, M. Ohnuma, B. Deconihout, K. Hono, Acta Mater. 52 (2004) 4427.

[32] M.W. Chen, A. Inoue, W. Zhang, T. Sakurai, Phys. Rev. Lett. 96 (2006) 245502.

[33] J.-J. Kim, Y. Choi, S. Suresh, A.S. Argon, Science 295 (2002) 654.

[34] G. Wilde, H. Rösner, Appl. Phys. Lett. 98 (2011) 251904. 
[35] X.J. Liu, Y. Xu, X. Hui, Z.P. Lu, F. Li, G.L. Chen, J. Lu, C.T. Liu, Phys. Rev. Lett. 105 (2010) 155501.

[36] A. Hirata, P.F. Guan, T. Fujita, Y. Hirotsu, A. Inoue, A.R. Yavari, T. Sakurai, M.W. Chen, Nat. Mater. 10 (2011) 28.

[37] R. Maranganti, P. Sharma, Phys. Rev. Lett. 98 (2007) 195504.

[38] H. Eyring, J. Chem. Phys. 4 (1936) 283.

[39] M.H. Cohen, D. Turnbull, J. Chem. Phys. 31 (1959) 1164.

[40] F. Spaepen, Acta Metall. 25 (1977) 407.
[41] H. Gleiter, Acta Mater. 56 (2008) 5875.

[42] J.D. Bernal, J. Mason, Nature 188 (1960) 910.

[43] G. Knuyt, L. De Schepper, L.M. Stals, J. Phys. F: Met. Phys. 16 (1986) 1989.

[44] E. Pineda, Phys. Rev. B 73 (2006) 104109.

[45] M.Q. Jiang, S.Y. Jiang, L.H. Dai, Chin. Phys. Lett. 26 (2009) 106103.

[46] Q.-s. Zeng, Y. Ding, W.L. Mao, W. Yang, S.V. Sinogeikin, J. Shu, H.-k. Mao, J.Z. Jiang, Phys. Rev. Lett. 104 (2010) 105702. 